\title{
DETERMINANTS OF FISH CONSUMPTION IN OLDER PEOPLE: A COMMUNITY-BASED COHORT STUDY
}

\author{
A.T. Bakre', Y. Song', A. Clifford ${ }^{1}$, A. Chen ${ }^{3}$, T. Smith ${ }^{1}$, Y. Wan ${ }^{1}$, L. Devlin ${ }^{1}$, J. Jie Tang ${ }^{4}$, W. Zhou', \\ I.M. Danat ${ }^{1}$, Z.Hu ${ }^{5}$, R. Chen ${ }^{1}$
}

\begin{abstract}
Objectives: Habitual fish consumption and its determinants in older people have not been well investigated. We addressed these issues through a population-based cohort study. Methods: In 2001-2003 we interviewed a random sample of 3336 residents aged $\geq 60$ years in China, documenting socioeconomic status (SES) and disease risk factors. In 2007-2009 we re-interviewed 1757 survivors, additionally surveying average self-reported intake of fish over the past two years. Results: Of 1757 participants, 1697 responded to the fish consumption questionnaire; $23.0 \%$ of whom had "never eat" fish, 43.4\% "once a week", 26.9\% "more than twice a week", and $6.7 \%$ " $\geq$ once a day". There was an inverse association of fish consumption with older age (multivariate adjusted odds ratio 0.64 [95\% CI 0.45-0.92] and 0.35 [0.24-0.52] at ages of 75-79, and $\geq 80$ years), female gender (0.63, $0.47-0.84)$, smoking $(0.65,0.48-0.88)$, living in a rural area $(0.10,0.07-0.15)$, having educational level of $\leq$ primary school $(0.10$, 0.05-0.19), occupation of peasant $(0.08,0.05-0.14)$, low income $(0.11,0.07-0.18)$, financial difficulties $(0.25$, 0.18-0.34), being never married/divorced $(0.48,0.28-0.81)$, having undetected hypertension $(0.71,0.55-0.91)$, depression (0.50, 0.29-0.84) and dementia (0.64, 0.41-0.98). However, participants with central obesity and heart disease at baseline had increased odds of fish consumption. Separate data analysis for different levels of fish consumption showed a dose-response trend for these associations. Conclusion: In older Chinese, there are large socioeconomic inequalities, and certain lifestyle, psychosocial factors and health-related conditions are strong determinants of fish consumption. Such information is important for future development or refinement of effective dietary interventions targeting older adults.
\end{abstract}

Key words: Fish consumption, risk factors, older adults.

\section{Introduction}

Globally, fish consumption has contributed immensely to the health of the people by reducing their morbidities and mortality (1). Its consumption has been associated with a decreased risk of cardiovascular diseases (CVD) (2). Fish contains essential nutrients, including vitamins, minerals and amino acids $(1,3,4)$, which makes it generally accepted as a vital component of a healthy and balanced diet (5). It is a significant source of animal protein that contains essential nutrients among which are long chain omega-3 polyunsaturated fatty acids (6), that assist in promoting the cognitive wellbeing of people $(7$, 8). Our recent study (9) showed that older people with increased consumption of fish had a reduced risk of dementia. Fish consumption in older age benefits late-life

1. Faculty of Education, Health and Wellbeing, University of Wolverhampton, UK, 2. Richard M. Fairbanks School of Public Health, Indiana University Indianapolis, Indiana, US; 3. Faculty of Sciences and Technology, Middlesex University, UK; 4. School of Public Health, Guangzhou Medical University, China; 5. School of Health Administrations, Anhui Medical University, China

Corresponding Author: Professor Ruoling Chen, Centre for Health and Social Care Improvement (CHSCI), University of Wolverhampton, Wolverhampton, WV1 1DT, UK. T: +44 (0)1902 328622, F: +44 (0)1902 321161, E: r.chen@wlv.ac.uk quality (10) and reduces the risks of neurodegenerative disorders (11) and all-cause mortality $(12,13)$. However, many older people reduce their fish consumption or do not eat fish at all. Existing literature $(14,15)$ shows that older people eat less fish than young and middleage populations though the reasons for this are unclear. Few studies have examined factors influencing the consumption of fish in older people, despite the world's population aging. Therefore, this study was conducted to examine the data from a population-based cohort to identify the determinants of fish consumption in older people which may help to increase fish consumption in the aging population.

\section{Methods}

\section{Study Participants}

The study population was derived from the Anhui cohort study. The methods of the Anhui cohort study have been fully described elsewhere (16). In brief, we randomly recruited 1810 people over 65 years old who had lived more than five years in Yiming subdistrict of Hefei city in $2001(17,18)$ and 1709 over 60 years old from 
all 16 villages in Tangdian district of Yingshang county in 2003 (19). In total 3336 adults agreed to participate in the present study (response rate of $94.8 \%$ ), of whom 1736 were living in urban and 1600 in rural area. They were interviewed by a trained survey team from the Anhui Medical University. Permission for interview and written informed consent were obtained from each participant. In about $5 \%$ of participants who could not provide informed consent, their nearest relative or carer were approached to provide assent to participation. The interview was conducted using the general health and risk factor record and the Geriatric Mental State (GMS) questionnaire (Wave 1) $(17,19)$. Participants' socio-demographic characteristics that comprise of their educational attainment, occupational class, level of income, financial status over the last two years, lifestyle, social networks and support, histories of chronic diseases and risk factors were recorded. Participants' anthropometric data and blood pressure were also measured. Participants' dementia and depression status were diagnosed using the Geriatric Mental StateAutomated Geriatric Examination for Computer Assisted Taxonomy (GMS-AGECAT) data (17). At one year after baseline, the interview team re-examined 2806 surviving participants (Wave 2), using the same protocol as before (20). In 2007-2009 (6 years after baseline), 1757 survivors were successfully re-interviewed (Wave 3) (16) and information about their dietary intakes of rice, wheat flour, meat, fish, egg, fresh vegetable, fruit, chilli pepper, garlic, ginger and different types of vegetable oils were collected. Participants' frequency of fish intake in the past two years was recorded as (1) Never eat, (2) $\leq$ Once a week, (3) > Once a week and $<$ Daily, (4) Once a day, and (5) $\geq$ Twice a day.

\section{Data Analysis}

We examined distributions of baseline risk factors and health conditions among participants with different levels of fish consumption documented at Wave 3 survey by chi-squared test for categorical variables and one-way analysis of variance for continuous outcome variables. We employed binomial logistic regression models to examine the determinants of older people having any level of fish consumption over the past two years versus those whose stated they "never eat" fish. We calculated the odds ratio (OR) and 95\% confidence intervals of each baseline risk factor associated with the consumption of fish in a 6-year follow up. In the models, we adjusted for age and sex first, to compute the OR. We further examined those variables that were significant in the age-sex adjusted analysis, with multivariate adjustment including waist circumference and smoking at the baseline. Finally, we analysed the data of different levels of fish consumptions respectively versus those who reported they "never eat" fish in the multivariate adjusted logistic regression models to investigate any trend in the associations of baseline risk factors with consumption of fish. All data analysis was performed using SPSS version 20 (SPSS Inc., Chicago, IL).

\section{Results}

Of 1757 surviving participants, 1697 (96.6\%) responded to the fish consumption questionnaire. The average age (s.d.) of participants was 71.8 (6.9) years, and $53.8 \%$ were women. With respect to the past two years there were $390(23.0 \%)$ participants who reported they "never eat" fish, $737(43.4 \%)$ who consumed fish "once a week", $457(26.9 \%)$ "more than twice a week", and $113(6.7 \%)$ " $\geq$ once a day". Table 1 shows characteristics of participants across different fish consumption categories. Participants with increased consumption of fish were significantly more likely to be younger (except for participants aged 60-64 years, who were from rural areas only), not smoking and urban living, and to have larger waist circumference, high levels of education, occupational class and income, no financial difficulty, and high satisfaction of life at baseline. High level of fish consumption was significantly associated with being currently married, less frequently visiting children/relatives/neighbours, having help available when needed, and having normal blood pressure/ controlled hypertension, hypercholesterolemia, diabetes and heart disease, but not depression and dementia. There were no significant differences in drinking alcohol, contacting friends in the community and activity of daily living (ADL) score $(0-\geq 5)$ across four groups of fish consumption.

Table 2 shows numbers and age-sex adjusted ORs and 95\% CIs of having any level of fish consumption vs. "never eat". The patterns of distributions of these baseline risk factors between combining any levels of fish consumption and "never eat" were similar to those in Table 1. After adjustment for age and sex, significantly reduced odds of eating fish were found in older people with increased age (except for 60-64 years), smoking, rural living, low levels of education, occupation and income, financial difficulties and low satisfaction of life at baseline. The reduced odds were also found in those who had never married or divorced, visited children or other relatives daily, and had undetected hypertension, depression or dementia. But older people classified as overweight (BMI) and/or having central obesity (WC), heart disease and hypercholesterolemia at baseline had an increased consumption of fish.

In the multivariate adjusted analysis (Table 3 ), the significantly reduced odds of having any levels of fish consumption were observed in older people with increased age, female gender, low socio-economic status, financial difficulties and low satisfaction of life, had never married or divorced, and had undetected hypertension, depression and dementia. Having central obesity and heart disease at baseline was significantly associated with 
Table 1

Characteristics of participants with different fish consumption levels

\begin{tabular}{|c|c|c|c|c|c|}
\hline Variables & Never eat $\mathbf{n}(\%)$ & Once a weekn (\%) & More than twice a week $\mathbf{n}(\%)$ & $\geq$ Once a day $\mathrm{n}(\%)$ & P value \\
\hline \multicolumn{6}{|l|}{ Demographic factors } \\
\hline \multicolumn{6}{|l|}{ Age (years) } \\
\hline $60-64$ & $96(24.6)$ & $147(19.9)$ & $42(9.2)$ & $8(7.1)$ & \multirow[t]{5}{*}{$<0.001$} \\
\hline $65-69$ & $81(20.8)$ & $206(28.0)$ & $169(37.0)$ & $45(39.8)$ & \\
\hline $70-74$ & $83(21.3)$ & $177(24.0)$ & $141(30.9)$ & $40(35.4)$ & \\
\hline 75-79 & $68(17.4)$ & $135(18.3)$ & $73(16.0)$ & $16(14.2)$ & \\
\hline$\geq 80$ & $62(15.9)$ & $72(9.8)$ & $32(7.0)$ & $4(3.5)$ & \\
\hline \multicolumn{6}{|l|}{ Sex } \\
\hline Men & $176(45.1)$ & $320(43.4)$ & $231(50.5)$ & $57(50.4)$ & \multirow[t]{2}{*}{0.08} \\
\hline Women & $214(54.9)$ & $417(56.6)$ & $226(49.5)$ & $56(49.6)$ & \\
\hline \multicolumn{6}{|l|}{ BMI $(\mathrm{kg} / \mathrm{m} 2)$} \\
\hline$<20$ & $43(11.0)$ & $87(11.8)$ & $40(8.8)$ & $16(14.2)$ & \multirow[t]{4}{*}{0.15} \\
\hline $20-<23$ & $150(38.5)$ & $225(30.5)$ & $149(32.6)$ & $36(31.9)$ & \\
\hline $23-<26$ & $117(30.0)$ & $265(36.0)$ & $154(33.7)$ & $37(32.7)$ & \\
\hline$\geq 26$ & $80(20.5)$ & $160(21.7)$ & $114(24.9)$ & $24(21.2)$ & \\
\hline \multicolumn{6}{|l|}{ Waist Circumference $(\mathrm{cm})$} \\
\hline No Action & $267(68.5)$ & $456(61.9)$ & $232(50.8)$ & $54(47.8)$ & \multirow[t]{3}{*}{$<0.001$} \\
\hline Action Level 1 & $68(17.4)$ & $141(19.1)$ & $113(24.7)$ & $19(16.8)$ & \\
\hline Action Level 2 & $55(14.1)$ & $140(19.0)$ & $112(24.5)$ & $40(35.4)$ & \\
\hline \multicolumn{6}{|l|}{ Smoking over the last 2 years } \\
\hline No & $251(64.4)$ & $511(69.3)$ & $339(74.2)$ & $84(74.3)$ & \multirow[t]{2}{*}{0.01} \\
\hline Yes & $139(35.6)$ & $226(30.7)$ & $118(25.8)$ & $29(25.7)$ & \\
\hline \multicolumn{6}{|c|}{ Drinking alcohol over the last 2 years } \\
\hline No & $317(81.3)$ & $600(81.4)$ & $355(77.7)$ & $93(82.3)$ & \multirow[t]{2}{*}{0.38} \\
\hline Yes & $73(18.7)$ & $137(18.6)$ & $102(22.3)$ & $20(17.7)$ & \\
\hline \multicolumn{6}{|l|}{ Socioeconomic factor } \\
\hline \multicolumn{6}{|l|}{ Urban/rurality } \\
\hline Urban & $28(7.2)$ & $209(28.4)$ & $287(62.8)$ & $98(86.7)$ & \multirow[t]{2}{*}{$<0.001$} \\
\hline Rural & $362(92.8)$ & $528(71.6)$ & $170(37.2)$ & $15(13.3)$ & \\
\hline \multicolumn{6}{|l|}{ Educational level } \\
\hline >High 2nd School & $11(2.8)$ & $101(13.7)$ & $148(32.4)$ & $51(45.1)$ & \multirow[t]{4}{*}{$<0.001$} \\
\hline Secondary School & $12(3.1)$ & $68(9.2)$ & $87(19.0)$ & $26(23.0)$ & \\
\hline Primary School & $31(7.9)$ & $80(10.9)$ & $56(12.3)$ & $17(15.0)$ & \\
\hline Illiterate & $336(86.2)$ & $488(66.2)$ & $166(36.3)$ & $19(16.8)$ & \\
\hline \multicolumn{6}{|l|}{ Main occupation } \\
\hline $\begin{array}{l}\text { No formal job (including business/ } \\
\text { other/housewife) }\end{array}$ & $9(2.3)$ & $33(4.5)$ & $39(8.5)$ & $12(10.6)$ & \multirow[t]{4}{*}{$<0.001$} \\
\hline Official/teacher & $16(4.1)$ & $135(18.3)$ & $202(44.2)$ & $67(59.3)$ & \\
\hline Manual labourer & $10(2.6)$ & $63(8.5)$ & $59(12.9)$ & $19(16.8)$ & \\
\hline Peasant & $355(91.0)$ & $506(68.7)$ & $157(34.4)$ & $15(13.3)$ & \\
\hline \multicolumn{6}{|l|}{ Income satisfactory } \\
\hline Very satisfactory & $8(2.1)$ & $32(4.3)$ & $45(9.8)$ & $14(12.4)$ & $<0.001$ \\
\hline Satisfactory & $19(4.9)$ & $140(19.0)$ & $209(45.7)$ & $65(57.5)$ & \\
\hline Average & $16(4.1)$ & $41(5.6)$ & $28(6.1)$ & $17(15.0)$ & \\
\hline Poor & $347(89.0)$ & $524(71.1)$ & $175(38.3)$ & $17(15.0)$ & \\
\hline Financial difficulties over the last yea & & & & & \\
\hline No & $61(15.6)$ & $237(32.2)$ & $291(63.7)$ & $98(86.7)$ & $<0.001$ \\
\hline Yes & $329(84.4)$ & $500(67.8)$ & $166(36.3)$ & $15(13.3)$ & \\
\hline Satisfied with life/ current living & & & & & \\
\hline Very satisfactory & $179(45.9)$ & $329(44.6)$ & $143(31.3)$ & $22(19.5)$ & $<0.001$ \\
\hline Satisfactory & $162(41.5)$ & $341(46.3)$ & $276(60.4)$ & $78(69.0)$ & \\
\hline
\end{tabular}


Table 1 (continued)

Characteristics of participants with different fish consumption levels

\begin{tabular}{|c|c|c|c|c|c|}
\hline Variables & Never eat $\mathrm{n}(\%)$ & Once a weekn $(\%)$ & More than twice a week $\mathrm{n}(\%)$ & $\geq$ Once a day $\mathbf{n}(\%)$ & P value \\
\hline Poor & $2(0.5)$ & $4(0.5)$ & $3(0.7)$ & $2(1.8)$ & \\
\hline \multicolumn{6}{|c|}{ Social network and psychosocial factors } \\
\hline \multicolumn{6}{|l|}{ Marriage } \\
\hline Married & $270(69.2)$ & $554(75.2)$ & $364(79.6)$ & $94(83.2)$ & 0.01 \\
\hline Never married / Divorced & $26(6.7)$ & $33(4.5)$ & $9(2.0)$ & $2(1.8)$ & \\
\hline Widow & $94(24.1)$ & $150(20.4)$ & $84(18.4)$ & $17(15.0)$ & \\
\hline \multicolumn{6}{|c|}{ Frequency of visiting children or other relatives \& } \\
\hline Everyday & 28071.8) & $490(66.5)$ & $263(57.5)$ & $43(38.1)$ & $<0.001$ \\
\hline At least weekly & $71(18.2)$ & $143(19.4)$ & $131(28.7)$ & $42(37.2)$ & \\
\hline At least Monthly or less often & $27(6.9)$ & $81(11.0)$ & $52(11.4)$ & $24(21.2)$ & \\
\hline$<$ Yearly or Never & $12(3.1)$ & $23(3.1)$ & $11(2.4)$ & $4(3.5)$ & \\
\hline \multicolumn{6}{|l|}{ Help available when needed } \\
\hline No & $39(10.0)$ & $66(9.0)$ & $18(3.9)$ & $7(6.2)$ & 0.003 \\
\hline Yes & $351(90.0)$ & $671(91.0)$ & $439(96.1)$ & $106(93.8)$ & \\
\hline \multicolumn{6}{|l|}{ Cardiovascular disease and risk factors } \\
\hline \multicolumn{6}{|l|}{ Hypertension status } \\
\hline No hypertension $(<140 / 90 \mathrm{mmHg})$ & $162(41.5)$ & $313(42.5)$ & $188(41.1)$ & $55(48.7)$ & $<0.001$ \\
\hline Undetected & $172(44.1)$ & $269(36.5)$ & $130(28.4$ & $23(20.4$ & \\
\hline Untreated & $16(4.1)$ & $39(5.3)$ & $25(5.5)$ & $5(4.4)$ & \\
\hline Uncontrolled & $34(8.7)$ & $95(12.9)$ & $91(19.9)$ & $19(16.8)$ & \\
\hline Controlled & $6(1.5)$ & $21(2.8)$ & $23(5.0)$ & $11(9.7)$ & \\
\hline \multicolumn{6}{|l|}{ Hypercholesterolemia } \\
\hline No & $381(97.7)$ & $710(96.3)$ & $411(89.9)$ & $99(87.6)$ & $<0.001$ \\
\hline Yes & $9(2.3)$ & $26(3.5)$ & $40(8.8)$ & $13(11.5)$ & \\
\hline Unknown & 0 & $1(0.1)$ & $6(1.3)$ & $1(0.9)$ & \\
\hline \multicolumn{6}{|l|}{ Diabetes } \\
\hline No & $383(98.2)$ & $719(97.6)$ & $434(95.0)$ & $102(90.3)$ & $<0.001$ \\
\hline Yes & $7(1.8)$ & $16(2.2)$ & $23(5.0)$ & $10(8.8)$ & \\
\hline Unknown & 0 & $2(0.3)$ & 0 & $1(0.9)$ & \\
\hline \multicolumn{6}{|c|}{ Heart diseases (ischaemic, valve disease or others) } \\
\hline No & $369(94.6)$ & $666(90.4)$ & $369(80.7)$ & $95(84.1)$ & $<0.001$ \\
\hline Yes & $21(5.4)$ & $69(9.4)$ & $86(18.8)$ & $18(15.9)$ & \\
\hline Unknown & 0 & $2(0.3)$ & $2(0.4)$ & 0 & \\
\hline \multicolumn{6}{|l|}{ Activity of daily living (score) } \\
\hline 0 & $377(96.7)$ & $705(95.7)$ & $430(94.1)$ & $109(96.5)$ & 0.67 \\
\hline $1-5$ & $8(2.1)$ & $21(2.8$ & $19(4.2$ & $3(2.7)$ & \\
\hline$\geq 5$ & $5(1.3)$ & $11(1.5)$ & $8(1.8)$ & $1(0.9)$ & \\
\hline \multicolumn{6}{|l|}{ Depression and dementia status } \\
\hline No & $271(69.5)$ & $560(76.0)$ & $363(79.4)$ & $93(82.3)$ & 0.006 \\
\hline \multicolumn{6}{|l|}{ Depression subcase } \\
\hline Depression case & $25(6.4)$ & $28(3.8)$ & $17(3.7)$ & $1(0.9)$ & \\
\hline \multicolumn{6}{|l|}{ Dementia subcase } \\
\hline Dementia case & $35(9.0)$ & $51(6.9)$ & $19(4.2)$ & $7(6.2)$ & \\
\hline
\end{tabular}


Table 2

Age-sex adjusted OR of participants who had consumed fish at any level over the past two years

\begin{tabular}{|c|c|c|c|c|c|c|c|}
\hline \multirow{2}{*}{$\begin{array}{l}\text { Variables } \\
\text { Demographic factors }\end{array}$} & $\begin{array}{c}\text { Any levels of fish } \\
\text { consumption } \\
\mathrm{N}=1307(\%)\end{array}$ & $\begin{array}{l}\text { Never eat } \\
\mathrm{N}=390(\%)\end{array}$ & $P$ value & $\begin{array}{c}\text { Age-sex } \\
\text { adjusted OR }\end{array}$ & \multicolumn{2}{|c|}{$95 \%$ CI } & \multirow[t]{2}{*}{ P-value } \\
\hline \multirow{2}{*}{\multicolumn{8}{|c|}{ Age (years) }} \\
\hline & & & & & & & \\
\hline $60-64$ & $197(15.1)$ & $96(24.6)$ & $<0.001$ & 0.40 & 0.28 & 0.56 & $<0.001$ \\
\hline $65-69$ & $420(32.1)$ & $81(20.8)$ & & Ref & & & \\
\hline $70-74$ & $358(27.4)$ & $83(21.3)$ & & 0.83 & 0.59 & 1.16 & 0.28 \\
\hline $75-79$ & $224(17.1)$ & $68(17.4)$ & & 0.63 & 0.44 & 0.91 & 0.01 \\
\hline$\geq 80$ & $108(8.3)$ & $62(15.9)$ & & 0.34 & 0.23 & 0.50 & $<0.001$ \\
\hline \multicolumn{8}{|l|}{ Sex } \\
\hline Men & $608(46.5)$ & $176(45.1)$ & & Ref & & & \\
\hline Women & $699(53.5)$ & $214(54.9)$ & 0.63 & 0.95 & 0.75 & 1.19 & 0.64 \\
\hline \multicolumn{8}{|l|}{ BMI (kg/m2) } \\
\hline$<20$ & $143(10.9)$ & $43(11.0)$ & 0.06 & 1.22 & 0.82 & 1.82 & 0.32 \\
\hline $20-<23$ & $410(31.4)$ & $150(38.5)$ & & Ref & & & \\
\hline $23-<26$ & $456(34.9)$ & $117(30.0)$ & & 1.37 & 1.03 & 1.81 & 0.03 \\
\hline$\geq 26$ & $298(22.8)$ & $80(20.5)$ & & 1.31 & 0.96 & 1.80 & 0.09 \\
\hline \multicolumn{8}{|l|}{ Waist Circumference $(\mathrm{cm})$} \\
\hline No Action & $742(56.8)$ & $267(68.5)$ & $<0.001$ & & & Ref & \\
\hline Action Level 1 & $273(20.9)$ & $68(17.4)$ & & 1.47 & 1.07 & 2.03 & 0.02 \\
\hline Action Level 2 & $292(22.3)$ & $55(14.1)$ & & 1.95 & 1.38 & 2.75 & $<0.001$ \\
\hline \multicolumn{8}{|l|}{ Smoking over the last 2 years } \\
\hline No & $934(71.5)$ & $251(64.4)$ & 0.007 & & & & \\
\hline Yes & $373(28.5)$ & $139(35.6)$ & Ref & 0.62 & 0.46 & 0.83 & 0.001 \\
\hline \multicolumn{8}{|l|}{ Drinking alcohol over the 2 years } \\
\hline No & $1048(80.2)$ & $317(81.3)$ & 0.63 & & Ref & & \\
\hline Yes & $259(19.8)$ & $73(18.7)$ & & 1.03 & 0.75 & 1.41 & 0.86 \\
\hline \multicolumn{8}{|l|}{ Socioeconomic factor } \\
\hline \multicolumn{8}{|l|}{ Urban/rurality } \\
\hline Urban & $594(45.4)$ & $28(7.2)$ & $<0.001$ & & & Ref & \\
\hline Rural & $713(54.6)$ & $362(92.8)$ & & 0.10 & 0.07 & 0.15 & $<0.001$ \\
\hline \multicolumn{8}{|l|}{ Educational level } \\
\hline$\geq$ High 2nd School & $300(23.0)$ & $11(2.8)$ & $<0.001$ & & Ref & & \\
\hline Secondary School & $181(13.8)$ & $12(3.1)$ & & 0.56 & 0.24 & 1.29 & 0.17 \\
\hline Illiterate/ Primary School & $826(63.2)$ & $367(94.1)$ & & 0.09 & 0.05 & 0.17 & $<0.001$ \\
\hline \multicolumn{8}{|l|}{ Main occupation } \\
\hline $\begin{array}{l}\text { No formal job (including business/other/ } \\
\text { housewife) }\end{array}$ & $84(6.4)$ & $9(2.3)$ & $<0.001$ & 0.37 & 0.16 & 0.86 & 0.02 \\
\hline Official / teacher & $404(30.9)$ & $16(4.1)$ & & & Ref & & \\
\hline Manual labourer & $141(10.8)$ & $10(2.6)$ & & 0.55 & 0.24 & 1.25 & 0.15 \\
\hline Peasant & $678(51.9)$ & $355(91.0)$ & & 0.08 & 0.05 & 0.14 & $<0.001$ \\
\hline \multicolumn{8}{|l|}{ Income satisfactory } \\
\hline Very satisfactory & $91(7.0)$ & $8(2.1)$ & $<0.001$ & 0.53 & 0.22 & 1.25 & 0.15 \\
\hline Satisfactory & $414(31.7)$ & $19(4.9)$ & & Ref & & & \\
\hline Average & $86(6.6)$ & $16(4.1)$ & & 0.25 & 0.12 & 0.51 & $<0.001$ \\
\hline Poor & $716(54.8)$ & $347(89.0)$ & & 0.10 & 0.06 & 0.17 & $<0.001$ \\
\hline \multicolumn{8}{|l|}{ Financial difficulties over the last years } \\
\hline No & $626(47.9)$ & $61(15.6)$ & $<0.001$ & & & Ref & \\
\hline Yes & $681(52.1)$ & $329(84.4)$ & & 0.23 & 0.17 & 0.31 & $<0.001$ \\
\hline Satisfied with life/ current living & & & & & & & \\
\hline Very satisfactory & $494(37.8)$ & $179(45.9)$ & 0.001 & & 0.71 & 0.55 & 0.91 \\
\hline Satisfactory & $695(53.2)$ & $162(41.5)$ & & Ref & & & \\
\hline Average/ Poor & $118(9.0)$ & 49 (12.6) & & 0.55 & 0.38 & 0.81 & 0.002 \\
\hline
\end{tabular}


Table 2 (continued)

Age-sex adjusted OR of participants who had consumed fish at any level over the past two years

\begin{tabular}{|c|c|c|c|c|c|c|c|}
\hline \multirow{2}{*}{$\begin{array}{l}\text { Variables } \\
\text { Never married/ Divorced }\end{array}$} & \multirow{2}{*}{$\begin{array}{c}\begin{array}{c}\text { Any levels of fish } \\
\text { consumption } \\
\text { N=1307 (\%) }\end{array} \\
44(3.4)\end{array}$} & \multirow{2}{*}{$\begin{array}{c}\text { Never eat } \\
\mathbf{N}=390(\%)\end{array}$} & \multirow[t]{2}{*}{$P$ value } & \multirow{2}{*}{$\begin{array}{c}\begin{array}{c}\text { Age-sex } \\
\text { adjusted OR }\end{array} \\
0.46\end{array}$} & \multicolumn{2}{|c|}{$95 \% \mathrm{CI}$} & \multirow{2}{*}{$\begin{array}{r}\text { P-value } \\
0.004\end{array}$} \\
\hline & & & & & 0.27 & 0.78 & \\
\hline Widow & $251(19.2)$ & $94(24.1)$ & & 0.78 & 0.58 & 1.03 & 0.08 \\
\hline \multicolumn{8}{|c|}{$\begin{array}{l}\text { Frequency of visiting children or other } \\
\text { relatives }\end{array}$} \\
\hline Everyday & $796(60.9)$ & $280(71.8)$ & 0.001 & 0.71 & 0.53 & 0.96 & 0.03 \\
\hline At least weekly & $316(24.2)$ & $71(18.2)$ & & Ref & & & \\
\hline At least Monthly or less often & $157(12.0)$ & $27(6.9)$ & & 1.37 & 0.84 & 2.22 & 0.21 \\
\hline$<$ Yearly or Never & $38(2.9)$ & $12(3.1)$ & & 0.75 & 0.37 & 1.53 & 0.43 \\
\hline \multicolumn{8}{|l|}{ Contacting friends in the community } \\
\hline$<$ Yearly or Never & $62(4.7)$ & $23(5.9)$ & 0.58 & & 0.72 & 0.43 & 1.22 \\
\hline At least Monthly or less often & $310(23.7)$ & $97(24.9)$ & & 0.88 & 0.66 & 1.19 & 0.41 \\
\hline At least weekly & $527(40.3)$ & $144(36.9)$ & & Ref & & & \\
\hline Everyday & $408(31.2)$ & $126(32.3)$ & & 0.85 & 0.65 & 1.12 & 0.25 \\
\hline \multicolumn{8}{|l|}{ Contacting neighbours } \\
\hline$<$ Yearly or Never & $32(2.4)$ & $4(1.0)$ & 0.10 & & 2.00 & 0.69 & 5.81 \\
\hline At least Monthly or less often & $382(29.2)$ & $100(25.6)$ & & 1.13 & 0.85 & 1.52 & 0.40 \\
\hline At least weekly & $469(35.9)$ & $141(36.2)$ & & Ref & & & \\
\hline Everyday & $424(32.4)$ & $145(37.2)$ & & 0.87 & 0.66 & 1.14 & 0.31 \\
\hline \multicolumn{8}{|l|}{ Help available when needed } \\
\hline No & $91(7.0)$ & $39(10.0)$ & 0.05 & & Ref & & \\
\hline Yes & $1216(93.0)$ & $351(90.0)$ & & 1.42 & 0.95 & 2.13 & 0.09 \\
\hline \multicolumn{8}{|l|}{ Cardiovascular disease and risk factors } \\
\hline \multicolumn{8}{|l|}{ Hypertension status } \\
\hline No hypertension $(<140 / 90 \mathrm{mmHg})$ & $556(42.5)$ & $162(41.5)$ & $<0.001$ & & & Ref & \\
\hline Undetected & $422(32.3)$ & $172(44.1)$ & & 0.73 & 0.56 & 0.94 & 0.01 \\
\hline Untreated & $69(5.3)$ & $16(4.1)$ & & 1.18 & 0.66 & 2.11 & 0.57 \\
\hline Uncontrolled & $205(15.7)$ & $34(8.7)$ & & 1.59 & 1.06 & 2.39 & 0.03 \\
\hline Controlled & $55(4.2)$ & $6(1.5)$ & & 2.28 & 0.96 & 5.43 & 0.06 \\
\hline \multicolumn{8}{|l|}{ Hypercholesterolemia } \\
\hline No & $1220(93.3)$ & $381(97.7)$ & 0.004 & & Ref & & \\
\hline Yes & $79(6.0)$ & $9(2.3)$ & & 2.20 & 1.09 & 4.46 & 0.03 \\
\hline Unknown & $8(0.6)$ & 0 & & & & & \\
\hline \multicolumn{8}{|l|}{ Diabetes } \\
\hline No & $1255(96.0)$ & $383(98.2)$ & 0.104 & & Ref & & \\
\hline Yes & $49(3.7)$ & $7(1.8)$ & & 1.80 & 0.80 & 4.03 & 0.16 \\
\hline Unknown & $3(0.2)$ & 0 & & & & & \\
\hline \multicolumn{8}{|c|}{ Heart diseases (ischaemic, valve disease or others) } \\
\hline No & $1130(86.5)$ & $369(94.6)$ & $<0.001$ & & & Ref & \\
\hline Yes & $173(13.2)$ & $21(5.4)$ & & 2.48 & 1.55 & 3.98 & $<0.001$ \\
\hline Unknown & $4(0.3)$ & 0 & & & & & \\
\hline \multicolumn{8}{|l|}{ Activity of daily living (score) } \\
\hline 0 & $1244(95.2)$ & $377(96.7)$ & 0.42 & & Ref & & \\
\hline 1-5 & $43(3.3)$ & $8(2.1)$ & & 1.50 & 0.69 & 3.24 & 0.31 \\
\hline$\geq 5$ & $20(1.5)$ & $5(1.3)$ & & 1.26 & 0.46 & 3.46 & 0.65 \\
\hline \multicolumn{8}{|l|}{ Depression and dementia status } \\
\hline No & $1016(77.7)$ & $271(69.5)$ & .006 & & Ref & & \\
\hline Depression subcase & $43(3.3)$ & $14(3.6)$ & & 0.86 & 0.46 & 1.62 & 0.65 \\
\hline Depression case & $46(3.5)$ & $25(6.4)$ & & 0.49 & 0.29 & 0.83 & 0.01 \\
\hline Dementia subcase & $125(9.6)$ & 45 (11.5) & & 0.78 & 0.53 & 1.13 & 0.19 \\
\hline Dementia case & $77(5.9)$ & $35(9.0)$ & & 0.65 & 0.43 & 1.01 & 0.05 \\
\hline
\end{tabular}


increased fish consumption in the follow up.

Table 4 shows odds of fish consumption at "once a week", "more than twice a week" and " $\geq$ once a day" in relation to baseline risk factors, respectively. We found that there were similar patterns of ORs for these risk factors to those in their combinations (i.e. in any levels of fish consumption in Table 3). The findings in Table 4 revealed some trends in ORs across different levels of fish consumption. In the age group of $\geq 80$ years, a significantly reduced OR of fish consumption at "once a week", "more than twice a week" and " $\geq$ once a day" was $0.46,0.26$ and 0.12 , respectively. The matched figures in women were $0.85,0.39$ and 0.34 , in rural areas $0.20,0.05$ and 0.01, in financial difficulties $0.44,0.14$ and 0.04 ; all significant. Other factors (e.g. low education, occupation and income, smoking) showed similar trends in ORs with reduced level of fish consumption, except for heart disease and dementia (Table 4).

\section{Discussion}

Our population-based cohort study in China demonstrated that within an older population increased age, female gender, smoking, living in rural areas, low levels of education, occupation and income, financial difficulties, low life satisfaction, being never married/ divorced, and having undetected hypertension, depression and dementia were associated with reduced consumption of fish in late life. Older people who had central obesity or heart disease may have increased consumption of fish.

\section{Prevalence of fish consumption in older people}

Previous studies showed that compared to young people, older adults had a lower consumption of fish. In Turkey, Erdogan et al (21) found that the proportion of people eating fish twice a week at ages 41-50 years, $51-60$ years and $\geq 60$ years was $26.5 \%, 25.6 \%$ and $23.2 \%$ respectively. In a USA study of 932 current seafood consumers aged 65 years and above, $18.0 \%$ of older people consumed seafood two or more times/week (22). Our finding of $26.9 \%$ of older people consuming fish more than twice a week is therefore slightly higher than those in Turkey and USA, but less than reported in a cross-sectional study in France of 9280 participants aged $\geq 65$ years, where $44.1 \%$ had an intake of fish 2-3 times a week (23). Our results show that $43.4 \%$ of the participants consumed fish once a week, while Barberger-Gateau et al (23) reported a $38.4 \%$ fish intake of once a week among their French participants. The Anhui cohort study showed that $6.7 \%$ of older people consumed fish $\geq$ Once a day, while $6.3 \%$ daily or almost daily fish consumption was reported in Tanskanen et al (24) cross-sectional study of 3204 Finnish adults aged 25-64 years old. There is therefore variation in the amount of fish consumption in older people in different countries, probably due to income, culture and geographic place.

\section{Factors influencing the consumption of fish in older people}

\section{Age and Sex}

Our data of the Anhui cohort study shows that the odds of fish consumption decrease as age increases even within an older population. This is in accordance with an Australian cross-sectional study of 854 participants aged $\geq 51$ years old, which found an OR of 1.82 (1.202.75) for having $\geq 1 \frac{1}{2}$ serving of seafood per week among those aged 51-75 years when compared to those aged $\geq 76$ years (15). Larrieu et al (25) also reported infrequent fish consumption among older participants in a large population-based cross-sectional study of 9250 French older adults aged $\geq 65$ years. In a cross-sectional study of 127 randomly selected participants, Can et al (14) found that the annual fish consumption level of young people is almost double that of the older people. In contrast, in a Norway cross-sectional study of 9407 participants aged 45-69 years, Trondsen et al (26) observed that increase in age was associated with increased odds of fish consumption. Also, in a Belgium cross-sectional study examining 429 participants mean aged 40.6 years (age range $\leq 25->55$ ), Verbeke and Vackier's (27) found an increase in fish consumption level as age increases. The main literature indicates an inequality in fish consumption in older adults, although there are some inconsistent findings.

The lower odds of fish consumption found among females in this study was consistent with the findings of some previous studies. A Nigerian cross-sectional study of 210 participants aged 21-70 years also revealed a significant reduction in fish consumption level among the female participants (28). In Norway, examining a crosssectional study of 3144 participants aged 16-79 years, Johansson et al (29) found an increased daily intake of fish among their male participants. In Taiwan, $\mathrm{Li}$ et al (30) carried out a cross-sectional study of 1200 participants aged 14-71 years, and found a significantly reduced odds of fish consumption (OR 0.71) among female participants. However, in a Turkish study, Can et al (14) found that the females' yearly fish intake level was $1.19 \mathrm{~kg}$ more than the male participants' intake level. The differences among our Chinese study, and the three reported above $(28,29,30)$ in comparison with the Turkish study (14) could be due to some cultural differences or because women are more likely to be financially incapacitated, thereby making fish products very expensive to purchase, which in turn may impact on their frequency of fish consumption. 
Table 3

Multivariate adjusted $\mathrm{OR}^{*}$ of participants who had consumed fish at any level over the past two years

\begin{tabular}{lccc}
\hline Variables & $\begin{array}{c}\text { Adjusted } \\
\text { OR }^{*}\end{array}$ & $95 \%$ CI & P value \\
\hline
\end{tabular}

Demographic Factors

Age (years)

$60-64$
$65-69$
$70-74$
$75-79$
$\geq 80$
Sex
Men

Women

Waist Circumference $(\mathrm{cm})$

No Action

Action Level 1

Action Level 2

Smoking over the last 2 years

No

Yes

Socioeconomic factor

Urban/rurality

\section{Urban}

Rural

Educational level

$\geq$ High 2nd School

Secondary School

Illiterate/ Primary School

Main occupation

No formal job (including business/ other/housewife)

Official/teacher

Manual labourer

Peasant

Income satisfactory

Very satisfactory

Satisfactory

Average

Poor

Financial difficulties over the last years

$$
\text { No }
$$

Yes

Satisfied with life/ current living

Very satisfactory

Satisfactory

Average/ Poor

Marriage

Married

\begin{tabular}{|c|c|c|c|c|}
\hline Never married/ Divorced & 0.48 & 0.28 & 0.81 & 0.006 \\
\hline Widow & 0.79 & 0.59 & 1.05 & 0.10 \\
\hline \multicolumn{5}{|c|}{ Frequency of visiting children or other relatives\& } \\
\hline Everyday & 0.77 & 0.57 & 1.04 & 0.08 \\
\hline At least weekly & Ref & & & \\
\hline At least Monthly or less often & 1.39 & 0.85 & 2.27 & 0.19 \\
\hline$<$ Yearly or Never & 0.78 & 0.38 & 1.60 & 0.51 \\
\hline \multicolumn{5}{|l|}{ Help available when needed } \\
\hline No & Ref & & & \\
\hline Yes & 1.44 & 0.96 & 2.15 & 0.08 \\
\hline \multicolumn{5}{|l|}{ Cardiovascular disease and risk factors } \\
\hline \multicolumn{5}{|l|}{ Hypertension status } \\
\hline No hypertension $(<140 / 90 \mathrm{mmHg})$ & Ref & & & \\
\hline Undetected & 0.71 & 0.55 & 0.91 & 0.008 \\
\hline Untreated & 1.10 & 0.61 & 1.98 & 0.75 \\
\hline Uncontrolled & 1.34 & 0.88 & 2.04 & 0.17 \\
\hline Controlled & 1.89 & 0.79 & 4.54 & 0.16 \\
\hline \multicolumn{5}{|l|}{ Hypercholesterolemia } \\
\hline No & Ref & & & \\
\hline Yes & 1.87 & 0.92 & 3.82 & 0.09 \\
\hline \multicolumn{5}{|l|}{ Unknown } \\
\hline \multicolumn{5}{|c|}{ Heart diseases (ischaemic, valve disease or others) } \\
\hline No & Ref & & & \\
\hline Yes & 2.33 & 1.44 & 3.75 & 0.001 \\
\hline \multicolumn{5}{|l|}{ Unknown } \\
\hline \multicolumn{5}{|l|}{ Depression and dementia status } \\
\hline No & Ref & & & \\
\hline Depression subcase & 0.83 & 0.44 & 1.57 & 0.57 \\
\hline Depression case & 0.50 & 0.29 & 0.84 & 0.009 \\
\hline Dementia subcase & 0.73 & 0.50 & 1.07 & 0.10 \\
\hline Dementia case & 0.64 & 0.41 & 0.98 & 0.04 \\
\hline
\end{tabular}

* adjusted for age, sex, waist circumference and smoking at the baseline; \& Data for "Contacting friends in the community" and "Contacting neighbours" showed no significant ORs.

\section{Socioeconomic Status}

Educational level In a US cross-sectional study of 1062 participants aged 18 to over 65 years, Hick et al (22) found an increase in the frequency of seafood intake of two or more times a week among participants with higher educational level. Grieger et al's (15) Australian cross-sectional study of 854 participants found an increase in fresh finfish and canned fish consumption level among older participants aged $\geq 51$ years old with higher educational level. A French cross-sectional study showed an increase in frequency of fish consumption as educational level increases among participants aged $\geq 65$ years (23). The studies conducted by Can et al (14) and Anyanwu (28) in Turkey and Nigeria showed that people with low educational level had low level of fish consumption, which were consistent with the findings of our Anhui cohort study in China. But some other 
Table 4

Multivariate adjusted $\mathrm{OR}^{*}$ of participants who had different levels of fish consumption over the past two years

\begin{tabular}{|c|c|c|c|c|c|c|c|c|c|c|c|c|}
\hline \multirow[t]{2}{*}{ Variables } & \multicolumn{4}{|c|}{ Once a week } & \multicolumn{4}{|c|}{ More than twice a week } & \multicolumn{4}{|c|}{$>=$ Once a day } \\
\hline & $\begin{array}{l}\text { Adjusted } \\
\text { OR }^{*}\end{array}$ & \multicolumn{2}{|c|}{$95 \% \mathrm{CI}$} & P value & $\begin{array}{l}\text { Adjusted } \\
\text { OR }^{*}\end{array}$ & \multicolumn{2}{|c|}{$95 \% \mathrm{CI}$} & $P$ value & $\begin{array}{l}\text { Adjusted } \\
\text { OR }^{*}\end{array}$ & \multicolumn{2}{|c|}{$95 \% \mathrm{CI}$} & P value \\
\hline \multicolumn{13}{|l|}{ Demographic Factors } \\
\hline \multicolumn{13}{|l|}{ Age (years) } \\
\hline $60-64$ & 0.63 & 0.44 & 0.91 & 0.01 & 0.23 & 0.15 & 0.37 & $<0.001$ & 0.16 & 0.07 & 0.37 & $<0.001$ \\
\hline $65-69$ & Ref & & & & Ref & & & & Ref & & & \\
\hline $70-74$ & 0.86 & 0.59 & 1.24 & 0.41 & 0.80 & 0.54 & 1.18 & 0.26 & 0.87 & 0.50 & 1.52 & 0.63 \\
\hline $75-79$ & 0.78 & 0.53 & 1.15 & 0.21 & 0.53 & 0.34 & 0.82 & 0.004 & 0.42 & 0.21 & 0.84 & 0.01 \\
\hline$\geq 80$ & 0.46 & 0.30 & 0.71 & $<0.001$ & 0.26 & 0.15 & 0.44 & $<0.001$ & 0.12 & 0.04 & 0.35 & $<0.001$ \\
\hline \multicolumn{13}{|l|}{ Sex } \\
\hline Men & Ref & & & & Ref & & & & Ref & & & \\
\hline Women & 0.85 & 0.62 & 1.17 & 0.33 & 0.39 & 0.27 & 0.57 & $<0.001$ & 0.34 & 0.19 & 0.61 & $<0.001$ \\
\hline \multicolumn{13}{|l|}{ Waist Circumference $(\mathrm{cm})$} \\
\hline No Action & Ref & & & & Ref & & & & Ref & & & \\
\hline Action Level 1 & 1.14 & 0.81 & 1.63 & 0.46 & 2.08 & 1.39 & 3.09 & $<0.001$ & 1.34 & 0.69 & 2.61 & 0.38 \\
\hline Action Level 2 & 1.42 & 0.98 & 2.07 & 0.07 & 2.93 & 1.91 & 4.49 & $<0.001$ & 4.70 & 2.53 & 8.75 & $<0.001$ \\
\hline \multicolumn{13}{|l|}{ Smoking over the last 2 years } \\
\hline No & Ref & & & & Ref & & & & Ref & & & \\
\hline Yes & 0.79 & 0.57 & 1.08 & 0.14 & 0.51 & 0.35 & 0.74 & $<0.001$ & 0.43 & 0.24 & 0.77 & 0.004 \\
\hline \multicolumn{13}{|l|}{ Socioeconomic factor } \\
\hline \multicolumn{13}{|l|}{ Urban/rurality } \\
\hline Urban & Ref & & & & Ref & & & & Ref & & & \\
\hline Rural & 0.20 & 0.13 & 0.31 & $<0.001$ & 0.05 & 0.03 & 0.08 & $<0.001$ & 0.01 & 0.00 & 0.02 & $<0.001$ \\
\hline \multicolumn{13}{|l|}{ Educational level } \\
\hline$\geq$ High 2nd School & Ref & & & & Ref & & & & Ref & & & \\
\hline Secondary School & 0.65 & 0.27 & 1.58 & 0.35 & 0.55 & 0.23 & 1.30 & 0.17 & 0.62 & 0.23 & 1.66 & 0.34 \\
\hline Illiterate/Primary School & 0.19 & 0.10 & 0.36 & $<0.001$ & 0.06 & 0.03 & 0.12 & $<0.001$ & 0.03 & 0.01 & 0.07 & $<0.001$ \\
\hline \multicolumn{13}{|l|}{ Main occupation } \\
\hline $\begin{array}{l}\text { No formal job (including } \\
\text { business/other/housewife) }\end{array}$ & 0.43 & 0.17 & 1.06 & 0.07 & 0.36 & 0.15 & 0.89 & 0.03 & 0.38 & 0.13 & 1.12 & 0.08 \\
\hline Official/teacher & Ref & & & & Ref & & & & Ref & & & \\
\hline Manual labourer & 0.72 & 0.31 & 1.70 & 0.46 & 0.54 & 0.23 & 1.28 & 0.16 & 0.54 & 0.19 & 1.51 & 0.24 \\
\hline Peasant & 0.17 & 0.10 & 0.30 & $<0.001$ & 0.04 & 0.03 & 0.08 & $<0.001$ & 0.01 & 0.003 & 0.02 & $<0.001$ \\
\hline \multicolumn{13}{|l|}{ Income satisfactory } \\
\hline Very satisfactory & 0.57 & 0.23 & 1.43 & 0.23 & 0.51 & 0.21 & 1.25 & 0.14 & 0.58 & 0.20 & 1.66 & 0.31 \\
\hline Satisfactory & Ref & & & & Ref & & & & Ref & & & \\
\hline Average & 0.35 & 0.17 & 0.75 & 0.01 & 0.18 & 0.08 & 0.39 & $<0.001$ & 0.32 & 0.13 & 0.80 & 0.01 \\
\hline Poor & 0.23 & 0.14 & 0.38 & $<0.001$ & 0.06 & 0.04 & 0.10 & $<0.001$ & 0.02 & 0.01 & 0.04 & $<0.001$ \\
\hline \multicolumn{13}{|l|}{$\begin{array}{l}\text { Financial difficulties over } \\
\text { the last years }\end{array}$} \\
\hline No & Ref & & & & Ref & & & & Ref & & & \\
\hline Yes & 0.44 & 0.31 & 0.62 & $<0.001$ & 0.14 & 0.10 & 0.21 & $<0.001$ & 0.04 & 0.02 & 0.07 & $<0.001$ \\
\hline \multicolumn{13}{|l|}{$\begin{array}{l}\text { Satisfied with life / current } \\
\text { living }\end{array}$} \\
\hline Very satisfactory & 0.91 & 0.70 & 1.19 & 0.49 & 0.52 & 0.38 & 0.72 & $<0.001$ & 0.28 & 0.16 & 0.49 & $<0.001$ \\
\hline Satisfactory & Ref & & & & & & & & & & & \\
\hline
\end{tabular}


Table 4

Multivariate adjusted $\mathrm{OR}^{*}$ of participants who had different levels of fish consumption over the past two years

\begin{tabular}{|c|c|c|c|c|c|c|c|c|c|c|c|c|}
\hline \multirow[t]{2}{*}{ Variables } & \multicolumn{4}{|c|}{ Once a week } & \multicolumn{4}{|c|}{ More than twice a week } & \multicolumn{4}{|c|}{$>=$ Once a day } \\
\hline & $\begin{array}{l}\text { Adjusted } \\
\text { OR }^{*}\end{array}$ & \multicolumn{2}{|c|}{$95 \% \mathrm{CI}$} & P value & $\begin{array}{l}\text { Adjusted } \\
\text { OR }^{*}\end{array}$ & \multicolumn{2}{|c|}{$95 \% \mathrm{CI}$} & $P$ value & $\begin{array}{l}\text { Adjusted } \\
\text { OR }^{*}\end{array}$ & \multicolumn{2}{|l|}{$95 \% \mathrm{CI}$} & P value \\
\hline \multicolumn{13}{|l|}{ Marriage } \\
\hline Married & Ref & & & & & Ref & & & & & Ref & \\
\hline Never married/Divorced & 0.64 & 0.37 & 1.10 & 0.11 & 0.24 & 0.10 & 0.54 & 0.001 & 0.21 & 0.05 & 0.97 & 0.046 \\
\hline Widow & 0.80 & 0.59 & 1.09 & 0.16 & 0.82 & 0.57 & 1.18 & 0.29 & 0.76 & 0.41 & 1.41 & 0.38 \\
\hline \multicolumn{13}{|c|}{ Frequency of visiting children or other relatives\& } \\
\hline Everyday & 0.93 & 0.67 & 1.29 & 0.67 & 0.65 & 0.45 & 0.94 & 0.02 & 0.32 & 0.19 & 0.56 & $<0.001$ \\
\hline At least weekly & Ref & & & & Ref & & & & Ref & & & \\
\hline $\begin{array}{l}\text { At least Monthly or less } \\
\text { often }\end{array}$ & 1.58 & 0.93 & 2.67 & 0.09 & 1.02 & 0.57 & 1.83 & 0.95 & 1.50 & 0.70 & 3.19 & 0.30 \\
\hline$<$ Yearly or Never & 0.98 & 0.46 & 2.09 & 0.95 & 0.59 & 0.23 & 1.48 & 0.26 & 0.79 & 0.22 & 2.87 & 0.72 \\
\hline \multicolumn{13}{|l|}{ Help available when needed } \\
\hline No & Ref & & & & Ref & & & & Ref & & & \\
\hline Yes & 1.14 & 0.74 & 1.73 & 0.56 & 3.00 & 1.63 & 5.52 & $<0.001$ & 1.91 & 0.79 & 4.61 & 0.15 \\
\hline \multicolumn{13}{|c|}{ Cardiovascular disease and risk factors } \\
\hline \multicolumn{13}{|l|}{ Hypertension status } \\
\hline $\begin{array}{l}\text { No hypertension }(<140 / 90 \\
\mathrm{mmHg})\end{array}$ & Ref & & & & Ref & & & & Ref & & & \\
\hline Undetected & 0.80 & 0.61 & 1.05 & 0.11 & 0.60 & 0.43 & 0.84 & 0.003 & 0.34 & 0.19 & 0.61 & $<0.001$ \\
\hline Untreated & 1.17 & 0.63 & 2.18 & 0.62 & 0.96 & 0.47 & 1.99 & 0.92 & 0.79 & 0.25 & 2.49 & 0.68 \\
\hline Uncontrolled & 1.21 & 0.77 & 1.90 & 0.40 & 1.54 & 0.95 & 2.51 & 0.08 & 1.03 & 0.49 & 2.15 & 0.94 \\
\hline Controlled & 1.47 & 0.57 & 3.77 & 0.43 & 2.33 & 0.87 & 6.22 & 0.09 & 3.51 & 1.04 & 11.89 & 0.04 \\
\hline \multicolumn{13}{|l|}{ Hypercholesterolemia } \\
\hline No & Ref & & & & Ref & & & & Ref & & & \\
\hline Yes & 1.21 & 0.55 & 2.65 & 0.64 & 2.19 & 1.02 & 4.70 & 0.04 & 3.82 & 1.45 & 10.02 & 0.007 \\
\hline \multicolumn{13}{|l|}{ Unknown } \\
\hline \multicolumn{13}{|c|}{ Heart diseases (ischaemic, valve disease or others) } \\
\hline No & Ref & & & & Ref & & & & Ref & & & \\
\hline Yes & 1.75 & 1.05 & 2.93 & 0.03 & 3.22 & 1.89 & 5.49 & $<0.001$ & 2.95 & 1.39 & 6.27 & 0.005 \\
\hline \multicolumn{13}{|l|}{ Unknown } \\
\hline \multicolumn{13}{|l|}{ Depression and dementia status } \\
\hline No & Ref & & & & Ref & & & & Ref & & & \\
\hline Depression subcase & 1.02 & 0.53 & 1.97 & 0.95 & 0.39 & 0.15 & 1.05 & 0.06 & 0.93 & 0.28 & 3.08 & 0.90 \\
\hline Depression case & 0.51 & 0.29 & 0.90 & 0.02 & 0.50 & 0.25 & 1.01 & 0.05 & 0.07 & 0.01 & 0.54 & 0.01 \\
\hline Dementia subcase & 0.72 & 0.48 & 1.08 & 0.11 & 0.80 & 0.50 & 1.27 & 0.34 & 0.48 & 0.19 & 1.19 & 0.11 \\
\hline Dementia case & 0.71 & 0.45 & 1.12 & 0.14 & 0.42 & 0.23 & 0.78 & 0.006 & 0.71 & 0.29 & 1.75 & 0.45 \\
\hline
\end{tabular}

studies (27) did not show a significant association of educational level with fish consumption. Trondsen et al (26) did not observe any significant effect of educational level on fish consumption. In Turkey, Erdogan et al (21) examined 972 participants aged 20 to over 60 years and found that $89.6 \%$ of uneducated or primary school level participants consume seafood, more than the high school and university degree level participants with $80.8 \%$ and
$85.4 \%$ seafood consumption respectively. The variation in the findings of each of the studies could be due to cultural differences in motivations for fish consumption. Where populations are relatively wealthy, e.g. such as in the United States of America, fish consumption is a choice. In poorer countries, it might be about what is available, so it has less to do with education. Coastal areas may also have more access to fresh fish regardless of wealth. 
Income Jensen (31) emphasized that the level of income is a significant determinant of the purchasing power of consumers' food and services, which affect how food is purchased. Can et al (14) established in their study that income is a significant determinant of fish consumption. Barberger-Gateau et al (23) showed a significantly increase odds of fish consumption with increase in income level among regular fish consumers. These findings are consistent with the results of our study. Trondsen et al (26) and Anyanwu (28) stated that a significant increase in household size shows a positive increase in the consumption of fish, which may be associated with income. However, Adeniyi et al's (32) Nigerian cross-sectional study found that the higher the participants' level of income the less they spent on fish products, thereby reducing their level of fish intake. This could be due to a preference for other expensive sources of animal protein in some populations.

Occupational class In Taiwan, Li et al (30) demonstrated that odds of fish consumption were reduced among the participants who had blue collar occupations. Johansson et al (29) also established in their Norwegian cross-sectional study of 3144 participants aged 16-79 years that blue-collar workers had a reduced intake of very-long-chain omega-3 fatty acids, which is the main component of fish protein. Galobardes et al (33) in their community based study of 5696 Swiss adults aged 35 to 74 years, found a reduced consumption of fish among participants with manual or lower occupational class. Our study also showed reduced odds among the peasant, manual laborers and those with no formal occupation. The group of low occupational class may have low levels of education and income. Both low levels of education and income appear to reduce the consumption of fish in the population throughout the life course including in older people.

\section{Social network and support}

\section{Marriage}

Our Anhui cohort study showed reduced odds of fish consumption among the 'Never married/Divorced' participants. In a Taiwan cross-sectional study of participants aged 14-71 years, Li et al (30) found lower odds of fish consumption among the unmarried participants. Barberger-Gateau et al (23) also showed reduced odds of fish consumption among the divorced, widow or single participants. Tanskanen et al (24) observed a reduced intake of fish among the unmarried participants in their cross-sectional study of 3204 Finnish adults aged 25-64 years old. Thong et al.'s (34) crosssectional study of 966 French adults mean aged 42 years (age range 18-65) revealed that their single participants consumed seafood less frequently when compared to those living with family or partner. However, Can et al's (14) cross-sectional study revealed a significantly greater yearly fish intake $(1.52 \mathrm{~kg})$ in single compared to married participants. The differences among our Chinese study, and the four reported above $(23,24,30,34)$ in comparison with the Turkish study (14) could be because those who were never married/divorced had a lower household income, and they may have fewer children at home which influences the demand for fish consumption.

\section{Cardiovascular disease and risk factors}

Smoking: Our cohort study showed that older people who smoked would have a lower level of fish consumption. A Finnish cross-sectional study of 3204 adults aged 25-64 years old showed that participants who rarely consumed fish are more likely to smoke (24). In a Norwegian cross-sectional study of 3144 participants, a non-significant association was found between smoking habit and intake of very-long-chain omega-3 fatty acids (29). However, Trondsen et al (26) found a significantly increased consumption of fish with smoking in a crosssectional study in Norway. These conflicting findings may be influenced by associations between smoking and low socioeconomic status, as well as intentions to maintain healthy lifestyles.

Obesity: Previous cross-sectional studies reported that fish consumers of more than once a week are significantly less likely to be obese (BMI $\geq 30 \mathrm{~kg} / \mathrm{m} 2$ ) (23), while another found that participants that rarely consume fish are less likely to be obese (24). However, our cohort study showed that older people who were overweight/ obese (BMI $\geq 26 \mathrm{~kg} / \mathrm{m} 2$ ) at baseline may have increased consumption of fish. This may be due to high income in those with obesity in China.

Undetected hypertension: Barberger-Gateau et al (23) France cross-sectional study observed that older people who suffered from hypertension consume fish more frequently, but our study showed that those with undetected hypertension at baseline would have a reduced consumption of fish, probably because these people were unaware of their state of health.

$r$ study shows an increase in fish consumption level among participants with heart disease. It was consistent with the finding from Devadawson et al's (35) study of 1777 participants aged 25-75 years. Devadawson et al (35) acknowledged that $37 \%$ of the participants in the study consumed fish based on curing their heart disease. Previous studies showed that based on health recommendations women with heart disease would have increased consumption of fish (27). Erdogan et al (21) also stated that $84.47 \%$ of the 972 participants consumed seafood based on its importance to health. This is in line with Can et al's (14) result, where $62.5 \%$ of their participants consumed fish based on health reasons. Trondsen et al (26) confirmed that seafood consumption was influenced by its beneficial impact on health. 


\section{Mental Health}

Our result shows that older people with depression had a significant decrease in fish consumption level. This is consistent with Barberger-Gateau et al's (23) France cross-sectional study that reported a significant decrease in fish consumption level among their older participants with depressive symptoms. Tanskanen et al (24) observed in a large population-based study of Finnish adults that the tendency of developing depressive symptoms is significantly higher among infrequent fish consumers. A five years cohort study of 10,602 men from Northern Ireland and France aged 50-59 years found that higher depressive mood was associated with lower fish intake (36). Astorg et al's (37) cohort study of 13,017 French participants aged 35-60 years observed a significantly reduced risk of any depressive episode among higher consumers of fatty fish or intake of long-chain omega-3 polyunsaturated fatty acid (PUFA).

Previous studies showed a significant reduction in fish consumption among the older participants with lower cognitive performance (23). Few studies investigated whether people with dementia had a reduced consumption of fish. As far as we know, our cohort study is the first reporting that older people with dementia had a significantly reduced consumption of fish. The reductions in fish intake among older people with depression or dementia could be due to reduced ability of the participants to choose to cook fish or to purchase fish at a restaurant.

\section{Strengths and Limitations of the study}

The main strength of our study lies in its cohort design of identifying possible influencing factors for fish consumption in older population. Our study cohort consists of two random samples of urban and rural Chinese who experienced epidemiological transition with specific characteristics, and we collected data on as many risk factors as possible, including mental health status. These have helped us to identify the determinants of low consumption of fish in older people for prevention. Our study has limitations. Firstly, there may be a recall bias from participants regarding fish consumption level that occurred during the interview. This would attenuate the associations that we found. Secondly, more detailed information about which type of fish intake (e.g. preserved) was not recorded and thus we could not examine its consumption levels. Thirdly, the inability to adjust for total energy intake in our study due to its absence among the variables assessed might have impacted on the overall result. But the adjustment for body weight (waist circumference) in the model and the strong association (e.g. OR 0.10) ensured that our results are robust.

\section{Implication of the Study}

Our study offers an insight into how the nutritional status regarding the consumption of inadequate fish protein among older people can be affected by sociodemographic and health factors. There is evidence that no or inadequate consumption of fish could impact on their cognitive function and increase the risk of cardiovascular disease $(2,38)$ and dementia (9). This result can help the government in their public health policies decision making. This could assist in channeling their resources towards availability and affordability of fish among socio-economically-deprived older populations. Boosting the economy income level through job creation might also enhance their overall food intake level including fish consumption, since food cannot be eaten in isolation, thus having a positive impact on their health and well-being. Facilitating the preparation technique of fish could also ease the stress displayed during cooking through provision of readymade boneless fish products that is accessible to purchase in the market. Especially for the high-risk groups with inadequate consumption of fish including older people with depression and dementia, possibly improving their prognosis.

In conclusion, the findings from our community-based cohort study suggested that reduced consumption of fish in older people was significantly associated with a number of factors. Targeting these high-risk groups of older people with low educational level, low income level and living in a rural area for preventing low consumption of fish would increase their level of consumption.

Acknowledgements: The authors thank the participants and all who were involved in the Anhui cohort study in China. Financial support: Ruoling Chen thanks the Royal Society, the BUPA Foundation and Alzheimer's Research UK to provide research grants for the Research Programme of Depression and Dementia in China, collecting the Anhui cohort study data.

Conflict of interest: The authors have no conflict of interests to declare.

Ethical standard: The study adhered to the current ethical standard that involve human participants.

\section{References}

1. Food and Agricultural organisation of the United Nations (FAO) 2016. The State of World Fisheries and Aquaculture: Contributing to Food Security and Nutrition for All: In Brief. Internet: http://www.fao.org/3/a-i5798e.pdf (accessed 10 September 2016) (accessed 10 September 2016).

2. Larsson SC, Orsini N. Fish consumption and the risk of stroke: a doseresponse meta-analysis. Stroke 2011;42:3621-3.

3. Kawarazuka N. The contribution of fish intake, aquaculture, and small-scale fisheries to improving nutrition: A literature review: The World Fish Center Working Paper. Internet: http:/ / www.worldfishcenter.org/resource_centre/ WF_2590.pdf (accessed 1st March 2018).

4. Lund EK. Health benefits of seafood; is it just the fatty acids? Food Chem 2013;140:413-20.

5. Yaktine AL, Nesheim MC. Seafood choices: balancing benefits and risks National Academies Press, 2007.

6. Connor WE. Importance of n-3 fatty acids in health and disease. Am J Clin Nutr 2000;71:171S-5S.

7. Kalmijn S, van Boxtel MP, Ocke M, Verschuren WM, Kromhout D, Launer LJ. Dietary intake of fatty acids and fish in relation to cognitive performance at middle age. Neurology 2004;62:275-80.

8. van Gelder BM, Tijhuis M, Kalmijn S, Kromhout D. Fish consumption, n- 3 
fatty acids, and subsequent 5-y cognitive decline in elderly men: the Zutphen Elderly Study-. Am J Clin Nutr 2007;85:1142-7.

9. Bakre AT, Chen R, Khutan R, Wei L, Smith T, Qin G, Danat IM, Zhou W, Schofield P, Clifford A. Association between fish consumption and risk of dementia: a new study from China and a systematic literature review and meta-analysis. Public Health Nutr 2018;1-12.

10. Schiepers O, De Groot R, Jolles J, Van Boxtel M. Fish consumption, not fatty acid status, is related to quality of life in a healthy population. Prostaglandins, Leukotrienes and Essential Fatty Acids 2010;83:31-5.

11. Zhang Y, Chen J, Qiu J, Li Y, Wang J, Jiao J. Intakes of fish and polyunsaturated fatty acids and mild-to-severe cognitive impairment risks: a dose-response meta-analysis of 21 cohort studies. Am J Clin Nutr 2016;103:330-40.

12. Yamagishi K, Iso H, Date C, Fukui M, Wakai K, Kikuchi S, Inaba Y, Tanabe N, Tamakoshi A. Fish, $\omega-3$ Polyunsaturated Fatty Acids, and Mortality From Cardiovascular Diseases in a Nationwide Community-Based Cohort of Japanese Men and Women: The JACC (Japan Collaborative Cohort Study for Evaluation of Cancer Risk) Study. Journal of the American College of Cardiology 2008;52:988-96.

13. Zhang J, Sasaki S, Amano K, Kesteloot H. Fish consumption and mortality from all causes, ischemic heart disease, and stroke: an ecological study. Prev Med 1999;28:520-9.

14. Can MF, Günlü A, Can HY. Fish consumption preferences and factors influencing it. Food Science and Technology (Campinas) 2015;35:339-46.

15. Grieger JA, Miller M, Cobiac L. Knowledge and barriers relating to fish consumption in older Australians. Appetite 2012;59:456-63.

16. Chen R, Hu Z, Wei L, Wilson K. Socioeconomic status and survival among older adults with dementia and depression. Br J Psychiatry 2014;204:436-40.

17. Chen $\mathrm{R}, \mathrm{Hu} \mathrm{Z}$, Qin X, Xu X, Copeland JR. A community-based study of depression in older people in Hefei, China-the GMS-AGECAT prevalence, case validation and socio-economic correlates. Int J Geriatr Psychiatry 2004;19:407-13.

18. Chen R, Song Y, Hu Z, Brunner EJ. Predictors of diabetes in older people in urban China. PloS one 2012;7:e50957.

19. Chen R, Wei L, Hu Z, Qin X, Copeland JR, Hemingway H. Depression in older people in rural China. Arch Intern Med 2005;165:2019-25.

20. Chen R, Hu Z, Wei L, Qin X, McCracken C, Copeland JR. Severity of depression and risk for subsequent dementia: cohort studies in China and the UK. Br J Psychiatry 2008;193:373-7.

21. Erdogan BE, Mol S, Cosansu S. Factors influencing the consumption of seafood in Istanbul, Turkey. Turkish Journal of Fisheries and Aquatic Sciences 2011;11:631-9.

22. Hicks D, Pivarnik L, McDermott R. Consumer perceptions about seafood-an internet survey. Journal of Foodservice 2008;19:213-26.

23. Barberger-Gateau P, Jutand MA, Letenneur L, Larrieu S, Tavernier B, Berr C. Correlates of regular fish consumption in French elderly community dwellers: data from the Three-City study. Eur J Clin Nutr 2005;59:817-25.
24. Tanskanen A, Hibbeln JR, Tuomilehto J, Uutela A, Haukkala A, Viinamäki H, Lehtonen J, Vartiainen E. Fish consumption and depressive symptoms in the general population in Finland. Psychiatric Services 2001;52:529-31.

25. Larrieu S, Letenneur L, Berr C, Dartigues J, Ritchie K, Alperovitch A, Tavernier B, Barberger-Gateau P. Sociodemographic Differences in Dieitary Habits in a Population-Based Sample of Elderly Subjects: The 3C Study. J Nutr Health Aging 2004;8:497-502.

26. Trondsen T, Braaten T, Lund E, Eggen A. Health and seafood consumption patterns among women aged45-69 years. A Norwegian seafood consumption study. Food quality and preference 2004;15:117-28.

27. Verbeke W, Vackier I. Individual determinants of fish consumption: application of the theory of planned behaviour. Appetite 2005;44:67-82.

28. Anyanwu S. Quantitative analysis of fish consumption in rivers state, Nigeria. American Journal of Experimental Agriculture 2014;4:469.

29. Johansson L, Solvoll K, Bjørneboe GA, Drevon C. Intake of very-longchain n-3 fatty acids related to social status and lifestyle. Eur J Clin Nutr 1998;52:716.

30. Li H, Houston JE, Wang S, Lee H. Factors affecting consumer preferences for fish in Taiwan. International Institute of Fisheries Economics and Trade (IIFET) 2000 Proceedings 2001;1-7.

31. Jensen $\mathrm{HH}$. Changes in seafood consumer preference patterns and associated changes in risk exposure. Mar Pollut Bull 2006;53:591-8.

32. Adeniyi OR, Omitoyin S, Ojo O. Socio-economic determinants of consumption pattern of fish among households in Ibadan North Local Government Area of Oyo State, Nigeria. African journal of food, agriculture, nutrition and development 2012;12:6537-52.

33. Galobardes B, Morabia A, Bernstein MS. Diet and socioeconomic position: does the use of different indicators matter? Int J Epidemiol 2001;30:334-40.

34. Thong NT, Solgaard HS. Consumer's food motives and seafood consumption. Food Quality and Preference 2017;56:181-8.

35. Devadawson C, Jayasinghe C, Sivakanesan R. Socio-demographic factors and fish eating trends in Eastern community, Sri Lanka. Journal of Experimental Biology and Agricultural Sciences 2015;3:423-9.

36. Appleton KM, Woodside JV, Yarnell JW, Arveiler D, Haas B, Amouyel P, Montaye M, Ferrieres J, Ruidavets JB, Ducimetiere P, et al. Depressed mood and dietary fish intake: direct relationship or indirect relationship as a result of diet and lifestyle? J Affect Disord 2007;104:217-23.

37. Astorg P, Couthouis A, Bertrais S, Arnault N, Meneton P, Guesnet P, Alessandri J, Galan P, Hercberg S. Association of fish and long-chain n-3 polyunsaturated fatty acid intakes with the occurrence of depressive episodes in middle-aged French men and women. Prostaglandins, Leukotrienes and Essential Fatty Acids 2008;78:171-82.

38. He K, Song Y, Daviglus ML, Liu K, Van Horn L, Dyer AR, Goldbourt U, Greenland P. Fish consumption and incidence of stroke: a meta-analysis of cohort studies. Stroke 2004;35:1538-42. 